Article

\title{
Deep Convolutional Neural Network for Mapping Smallholder Agriculture Using High Spatial Resolution Satellite Image
}

\author{
Bin Xie ${ }^{1(\mathbb{D}, \text { Hankui K. Zhang }}{ }^{2, *(\mathbb{D})}$ and Jie Xue ${ }^{3}$ (D) \\ 1 Institute of Remote Sensing and Earth Sciences, Hangzhou Normal University, Hangzhou 311121, China; \\ xiebin@hznu.edu.cn \\ 2 Geospatial Sciences Center of Excellence, South Dakota State University, Brookings, SD 57007, USA \\ 3 Department of Geography and Resource Management, The Chinese University of Hong Kong, Shatin, \\ Hong Kong, China; jiexue@link.cuhk.edu.hk \\ * Correspondence: hankui.zhang@sdstate.edu; Tel.: +1-605-688-4225
}

Received: 19 April 2019; Accepted: 23 May 2019; Published: 25 May 2019

\begin{abstract}
In classification of satellite images acquired over smallholder agricultural landscape with complex spectral profiles of various crop types, exploring image spatial information is important. The deep convolutional neural network $(\mathrm{CNN})$, originally designed for natural image recognition in the computer vision field, can automatically explore high level spatial information and thus is promising for such tasks. This study tried to evaluate different CNN structures for classification of four smallholder agricultural landscapes in Heilongjiang, China using pan-sharpened $2 \mathrm{~m}$ GaoFen-1 (meaning high resolution in Chinese) satellite images. $\mathrm{CNN}$ with three pooling strategies: without pooling, with max pooling and with average pooling, were evaluated and compared with random forest. Two different numbers ( 70,000 and $\sim 290,000)$ of CNN learnable parameters were examined for each pooling strategy. The training and testing samples were systematically sampled from reference land cover maps to ensure sample distribution proportional to the reference land cover occurrence and included 60,000-400,000 pixels to ensure effective training. Testing sample classification results in the four study areas showed that the best pooling strategy was the average pooling $\mathrm{CNN}$ and that the CNN significantly outperformed random forest (2.4-3.3\% higher overall accuracy and $0.05-0.24$ higher kappa coefficient). Visual examination of $\mathrm{CNN}$ classification maps showed that $\mathrm{CNN}$ can discriminate better the spectrally similar crop types by effectively exploring spatial information. CNN was still significantly outperformed random forest using training samples that were evenly distributed among classes. Furthermore, future research to improve CNN performance was discussed.
\end{abstract}

Keywords: deep convolutional neural network (CNN); high spatial resolution; GaoFen-1; smallholder agriculture

\section{Introduction}

Land cover plays an important role in human-environment interactions [1-4] including nature resource management, precision agriculture, ecosystem service modelling, climate change and urban planning, and has been recognized as an essential climate variable by the Global Climate Observing System (GCOS). Land cover mapping was undertaken since the first acquisition of satellite images [5]. Over the last several decades, land cover mapping has evolved from low to high spatial resolution [6,7], from local to global scale [8,9] and from single to time series image utilization [10,11]. This is attributed to the advancements in open data policy [12], analysis ready data [13], and machine learning and computation facilities [4]. 
The state-of-the-art of remote sensing image classification is to use non-parametric supervised classifiers, e.g., support vector machine [14] and random forest [15], trained with collected land cover samples. However, these machine learning classifiers need hand-engineered features to achieve good performance. Recently, deep convolutional neural network (CNN), due to its capability to extract features from raw data (end-to-end training), has significant advantage [16] in natural image scene classification in the computer vision field and has been proven in land cover mapping [17-20] (Table 1). The deep CNN originates from deep neural network, which has been in conception for decades [21] but only trainable and applicable after recent breakthrough in understanding of deep neural network [22]. The deep CNN has better representative capability as it usually consists of several to hundreds of feature layers linked by millions of learnable parameters to map the input predictors to the output class labels [16]. Convolution in CNN is usually applied on an image patch rather than a single pixel so that the high level spatial features are extractable. A convolution layer is usually followed by pooling operation, which combines several nearby features to a unique feature, to reduce noise, improve CNN efficiency [23], and keep scale and translational invariance features [24]. The popular pooling operation is max or average pooling [23], i.e., taking the max or average value of the nearby features as the pooling output.

Deep CNN has been used for detecting anomalies [25] and weeds [26] in agricultural field and for crop specie recognition [27] among many other agricultural applications [28]. A few studies have used deep CNN for cropland classification with median $[29,30]$ and high $[31,32]$ spatial resolution satellite images. The agricultural landscape is known to be difficult to classify reliably [33-35] especially smallholder crop areas [36-38] with field size smaller than 5 ha [36]. The smallholder agricultural landscape is mainly distributed in developing countries including China [39,40]. To map these heterogonous landscapes, effectively exploring spatial information is the key as many different crop types with similar spectral pattern coexist. The $\mathrm{CNN}$ is good at exploring spatial information, but the CNN structure parameters need to be carefully tuned to maximize its performance. This is because the optimal CNN structure may depend on the satellite image characteristics, input image patch size and training sample characteristic, e.g., Table 1 lists the $\mathrm{CNN}$ structure variety in the literature for remote sensing image classification.

Table 1. Pooling operation, input image patch size and layer number in supervised convolutional neural network $(\mathrm{CNN})$ used in the literature for remote sensing image land cover classification. Only layers with learnable parameters are counted following Simonyan and Zisserman [41] (e.g., pooling operation is not a layer).

\begin{tabular}{|c|c|c|c|}
\hline Literatures & Pooling & Input Image Patch Size & Layer Number \\
\hline Chen et al. [42] & $\max$ & $27 \times 27$ & 7 \\
\hline Zhao and Du [43] & $\max$ & $32 \times 32$ & $1,2,3,4,5$ \\
\hline Kussul et al. [29] & $\max$ & $7 \times 7$ & 4 \\
\hline Guo et al. [44] & average & $18 \times 18$ & 4 \\
\hline Li et al. [45] & no & $5 \times 5$ & 4 \\
\hline Mei et al. [46] & no & $3 \times 3 ; 5 \times 5$ & 3 \\
\hline Santara et al. [47] & no & $3 \times 3$ & $5,6,7$ \\
\hline Yang et al. [48] & $\max$ & $21 \times 21$ & 4 \\
\hline Wu and Prasad [49] & $\max$ & $11 \times 11$ & 4 \\
\hline Hamida et al. [50] & $\max$ & $3 \times 3 ; 5 \times 5 ; 7 \times 7$ & $5,7,9,11$ \\
\hline Ji et al. [31] & average; $\max$ & $8 \times 8$ & 5 \\
\hline Xu et al. [51] & $\max$ & $7 \times 7 ; 9 \times 9 ; 11 \times 11$ & 4 \\
\hline Liu et al. [52] & $\max$ & $9 \times 9$ & 4 \\
\hline Song et al. [53] & average & $23 \times 23 ; 25 \times 25 ; 27 \times 27$ & $26 ; 32$ \\
\hline Hao et al. [54] & $\max$ & $7 \times 7$ & 5 \\
\hline
\end{tabular}


Table 1. Cont.

\begin{tabular}{cccc}
\hline Literatures & Pooling & Input Image Patch Size & Layer Number \\
\hline Zhong et al. [55] & average & $3 \times 3 ; 5 \times 5 ; 7 \times 7 ; 9 \times 9 ; 11 \times 11$ & 12 \\
\hline Zhang et al. [56] & max & $16 \times 16$ & 5 \\
\hline Yang et al. [57] & no & $7 \times 7$ & $5 ; 10$ \\
\hline Mahdianpari et al. [58] & max & $\begin{array}{c}30 \times 30 \text { and resampled to the input size for } \\
\text { each CNN designed in computer vision field }\end{array}$ & $16 \sim 152$ \\
\hline Gao et al. [59] & max & $5 \times 5 ; 7 \times 7 ; 9 \times 9$ & 4 \\
\hline Karakizi et al. [60] & max & $29 \times 29$ & 4 \\
\hline Wei et al. [61] & no & $3 \times 3$ & 9 \\
\hline Paoletti et al. [62] & average & $11 \times 11$ & 25 \\
\hline Wang et al. [63] & average & & 12 \\
\hline Li et al. [64] & maximum overlap & & 4
\end{tabular}

The objectives of this study are to (1) apply the deep CNN to classify high spatial resolution images acquired over smallholder agricultural landscapes and (2) to compare CNN with the established random forest classifier. This study uses pan-sharpened GaoFen- 1 images with $2 \mathrm{~m}$ spatial resolution over four areas in Heilongjiang, China with smallholder agriculture. The reference land cover maps are interpreted from $2 \mathrm{~m}$ GaoFen-1 images and systematically sampled for training and testing. The CNN structure parameters are tuned with different pooling strategies and with different complexities. The classification results are evaluated using conventional confusion matrix derived from the testing samples and visual comparison of the classification maps.

\section{Data}

\subsection{Study Areas}

The study areas are in Heilongjiang province, which is located in the northeast agricultural area of China (Figure 1). The agricultural land accounts for $83.5 \%$ of the $473,000 \mathrm{~km}^{2}$ province land area. Four study areas including Dangnai village in Dorbod Mongol autonomous county (study area 1), Jufu village in Qing'an county (study area 2), Jiuwang village in Qing'an county (study area 3) and Changhe village in Bin county (study area 4 ) were chosen. The study area 1 is mainly covered by wetland and maize, study areas 2 and 3 by rice, and study area 4 by maize.

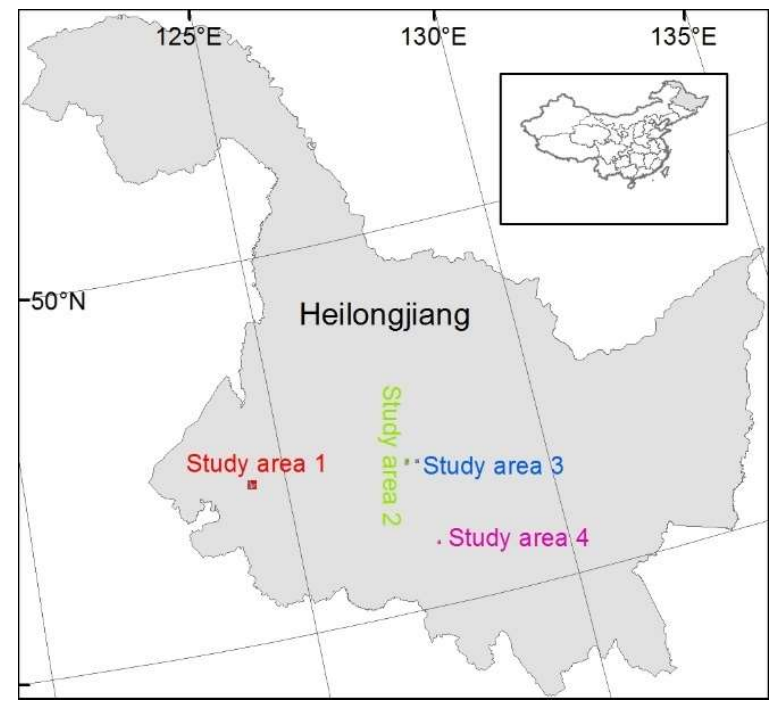

Figure 1. Four study area locations in Heilongjiang province, China. 


\subsection{GaoFen-1 Data}

The panchromatic/multispectral data from the China GaoFen-1 satellite were used in this study. The GaoFen-1 was launched in 2013 and provides 10-bit radiometric resolution panchromatic and multispectral data with 2 and $8 \mathrm{~m}$ spatial resolutions, respectively (Table 2). The detailed characteristics of GaoFen-1 data are summarized in Table 2.

Table 2. Characteristics of panchromatic/multispectral sensors aboard on the GaoFen-1 satellite.

\begin{tabular}{ccccc}
\hline Band & Wavelength $(\mathbf{n m})$ & Spatial Resolution $(\mathbf{m})$ & Re-Visiting Period (Days) & Swath (km) \\
\hline Panchromatic & $450-900$ & 2 & & \\
Blue & $450-520$ & 8 & & \\
Green & $520-590$ & 8 & 4 & 60 \\
Red & $630-690$ & 8 & & \\
Near infrared & $770-890$ & 8 & & \\
\hline
\end{tabular}

In this study, four GaoFen-1 panchromatic/multispectral images (Figure 2) over the four study areas were collected on 22 August, 13 August, 13 August and 17 August, 2016, respectively. All the $8 \mathrm{~m}$ four band multispectral images were pan-sharpened to $2 \mathrm{~m}$ using the well-established Gram-Schmidt method which is known to have moderate computational load and good spatial sharpening capability $[65,66]$. The images were mainly acquired in August considering that the harvest time of the main crops (wheat, rice, maize, etc.) in Northeast China is in September and October. They were subsequently cut according to the village administrative boundaries of the four study areas (Figure 2).

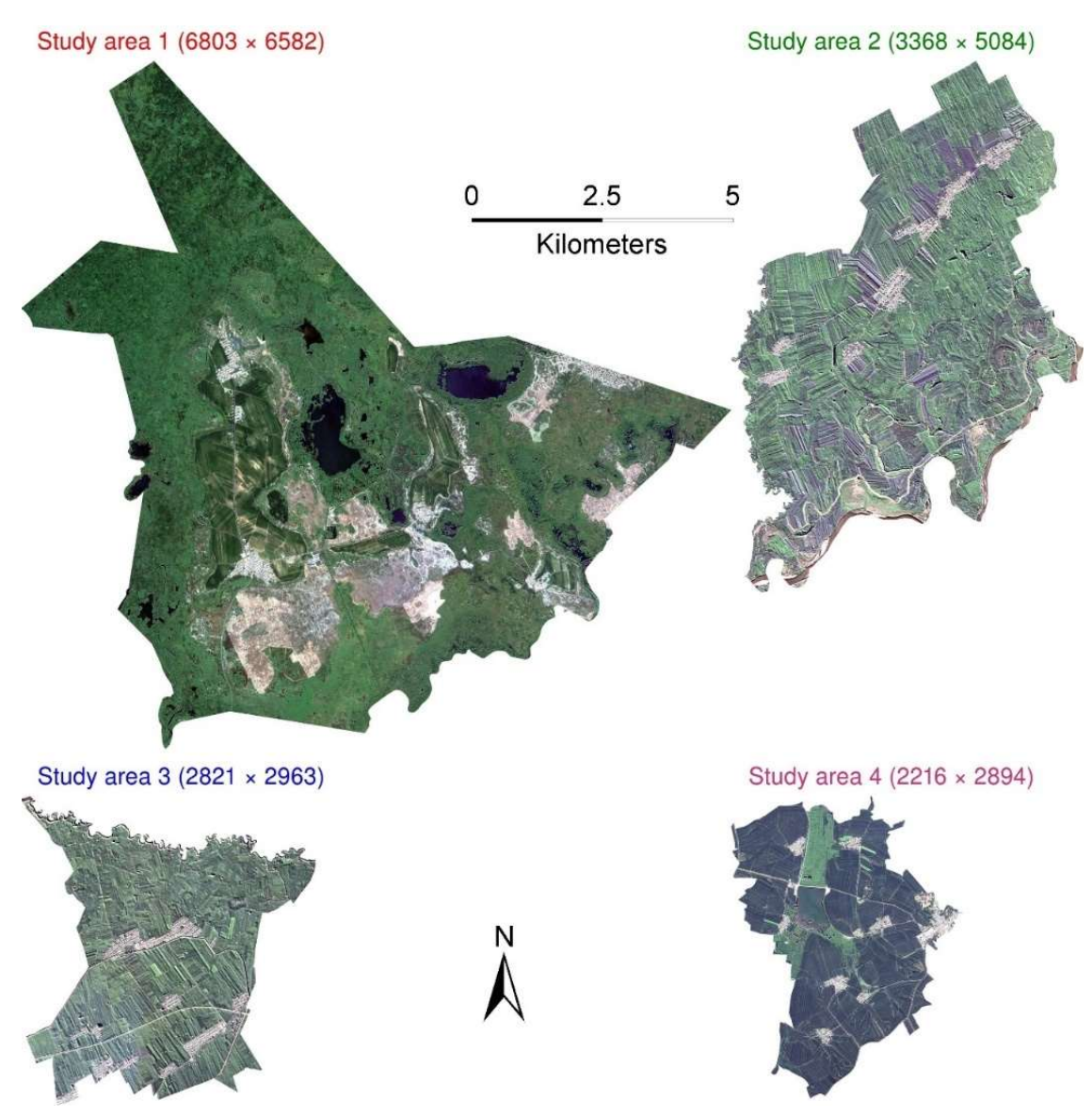

Figure 2. Four study area (locations shown in Figure 1) GaoFen-1 true color images with $6803 \times 6582$, $3368 \times 5084,2821 \times 2963$ and $2216 \times 28942 \mathrm{~m}$ pixels. Their sizes are proportional to their areas. 


\subsection{Reference Land Cover Maps for Training and Testing}

The reference land cover maps (Figure 3) were derived by visual interpretation of the $2 \mathrm{~m}$ pan-sharpened GaoFen-1 images with interpreter's field survey knowledge [67]. For each GaoFen-1 image, three sample plots each with $200 \times 200 \mathrm{~m}$ pixels were randomly selected and visited for land cover type identification by the interpreter in order to gain the necessary knowledge on the GaoFen-1 image characteristics of different land cover types. The entire study area image was then manually interpreted based on the knowledge. There are 14 land cover types including maize, rice, wheat, soybean, mung bean, vegetable, orchards, forest, grassland, water, wetland, road, residential and bare land. The small patch crop landscapes are evident in the reference landscapes (Figure 3).
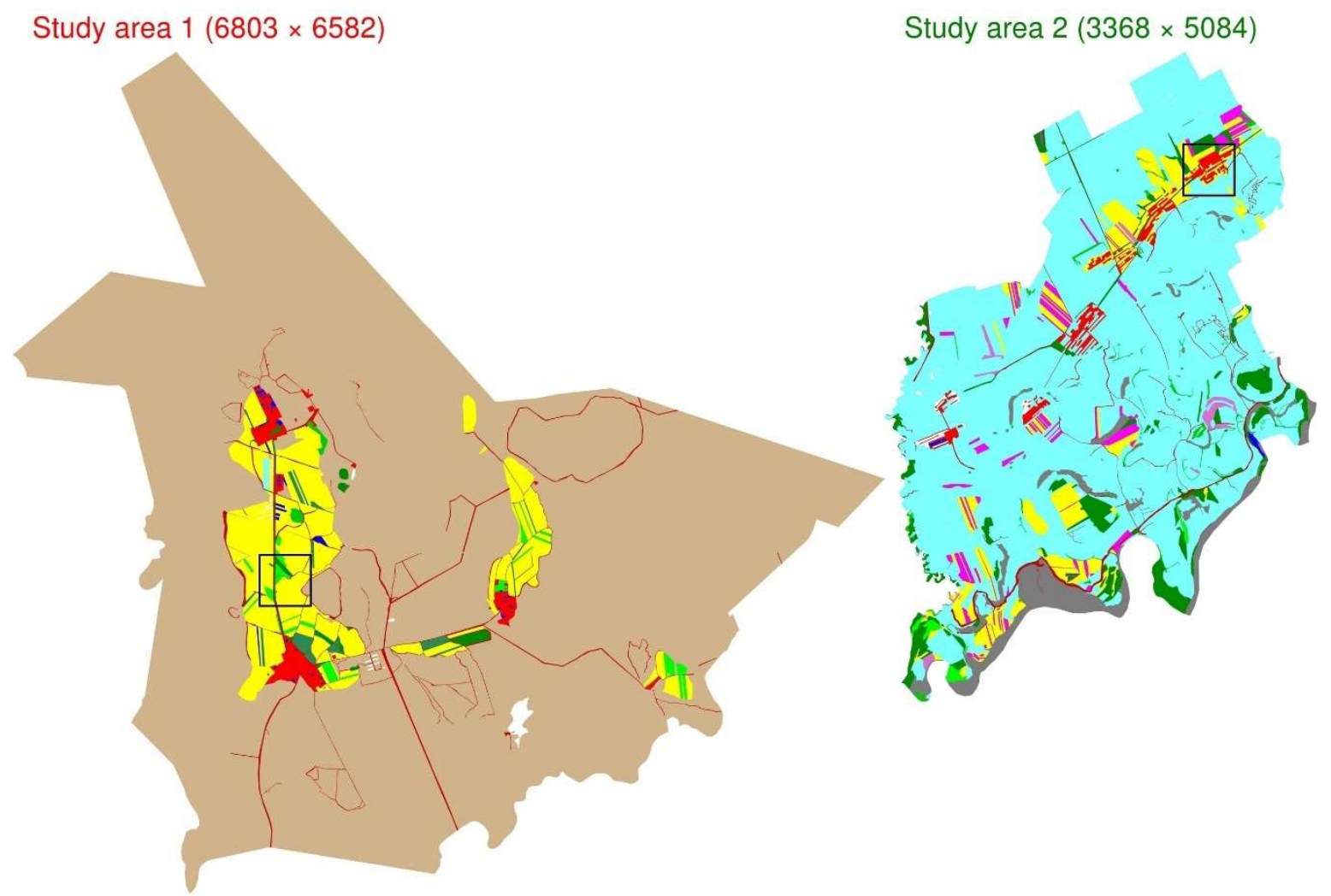

Study area $3(2821 \times 2963)$

Study area $4(2216 \times 2894)$
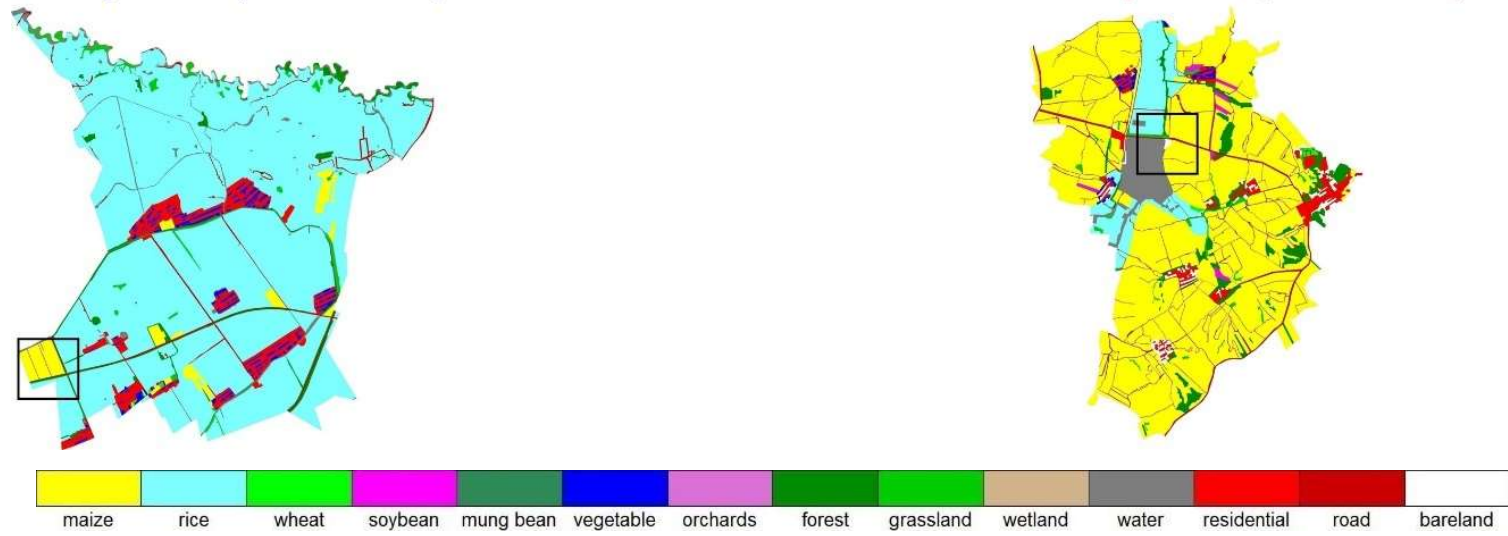

Figure 3. Four study area reference land cover maps (see Figure 2 caption for detailed information). The box covering $400 \times 4002 \mathrm{~m}$ pixels in each study area is examined in detail in the results section.

The training and testing samples were systematically collected from the reference land cover maps to make sure the proportional distribution among the classes related to the proportion that they 
occur in reality. Keeping class proportions in training samples has been shown with more reasonable classification accuracies $[10,68]$. The training and testing pixels cannot be very close to avoid that the spatial correlation may boost the testing data classification accuracy. They cannot be very far away to ensure enough training samples for the CNN model. Consequently, the reference land cover maps were sampled every five pixels in column and row directions. The sampled pixels in the pool were randomly and equally divided into training and testing samples. The training and testing sample numbers are shown in Table 3.

Table 3. Number of training and testing $2 \mathrm{~m}$ pixel samples in four study areas. The samples were derived by systematically sampling from the reference land cover maps (Figure 3 ) and randomly split into training and testing.

\begin{tabular}{ccccccccc}
\hline \multirow{2}{*}{ Classes } & \multicolumn{2}{c}{ Study Area 1 } & \multicolumn{2}{c}{ Study Area 2 } & \multicolumn{2}{c}{ Study Area 3 } & \multicolumn{2}{c}{ Study Area 4 } \\
\cline { 2 - 8 } & Training & Testing & Training & Testing & Training & Testing & Training & Testing \\
\hline Maize & 28,345 & 28,345 & 13,406 & 13,406 & 2574 & 2575 & 50,067 & 50,068 \\
Rice & 239 & 239 & 138,149 & 138,149 & 75,530 & 75,531 & 5396 & 5396 \\
Wheat & 1878 & 1879 & 720 & 721 & none & none & 139 & 139 \\
Soybean & none & none & 4458 & 4458 & none & none & 411 & 411 \\
Mung bean & 1419 & 1420 & none & none & none & none & none & none \\
Vegetable & 550 & 550 & 190 & 191 & 1505 & 1505 & 380 & 381 \\
Orchards & none & none & 1172 & 1173 & none & none & none & none \\
Forest & 1565 & 1565 & 9737 & 9737 & 2192 & 2192 & 3281 & 3281 \\
Grassland & 415 & 415 & 1325 & 1325 & 1048 & 1049 & 692 & 693 \\
Wetland & 352,942 & 352,943 & none & none & none & none & none & none \\
Water & none & none & 11,033 & 11,034 & 2080 & 2081 & 2915 & 2916 \\
Residential & 3677 & 3678 & 3205 & 3206 & 4348 & 4348 & 2121 & 2121 \\
Road & 5271 & 5271 & 2753 & 2753 & 1435 & 1435 & 3330 & 3331 \\
Bare land & 957 & 958 & 799 & 800 & none & none & 1038 & 1038 \\
Total & 397,258 & 397,263 & 186,947 & 186,953 & 90,712 & 90,716 & 69,770 & 69,775 \\
\hline
\end{tabular}

\section{Methods}

\subsection{CNN Overview}

CNN usually consists of multiple (several to more than one hundred) stacked layers each containing a certain number of features [16] (Figure 4) derived from the previous layer features by nonlinear transformation. Using these feature layers, the $\mathrm{CNN}$ is to convert the input $K \times K \times D$ ( $K$ being the spatial neighbor size and $D$ being the spectral band number, i.e., $D=4$ in this study) image patch to one single class label of the center pixel. Each layer feature is derived from the previous layer features through mathematical transformation, which nominally contains a linear operation with learnable weight and bias parameters and a fixed non-linear activation function. The convolution layer uses convolution kernel (the kernel values are the learnable weights) to extract information from a small region's neighbor pixels and contain many kernels to extract different feature information. The last convolution layer is usually followed by fully connected layers to extract more abstract features. These final, fully connected layer features are fed into a softmax function for classification. In such a way, the loss function can be defined using training samples with known land cover labels and the CNN training is to find the optimal learnable parameters to minimize the loss function. In this study, the cross-entropy loss function is used:

$$
\text { loss }=-\sum_{i=1}^{C}\left\{(y==i) * \log \frac{e^{a_{i}}}{\sum_{i=1}^{C} e^{a_{i}}}\right\},
$$

where $C$ is the total number of classes in the classification legend indexed by $i, y$ is the ground truth label, $a_{i}$ is the $i$ th feature value of the final fully connected layer with $C$ features. This loss function is 
minimized using a mini-batch gradient descent method where each gradient descent iteration only uses a small portion of the training samples to prevent overfitting and to save computation load [69].

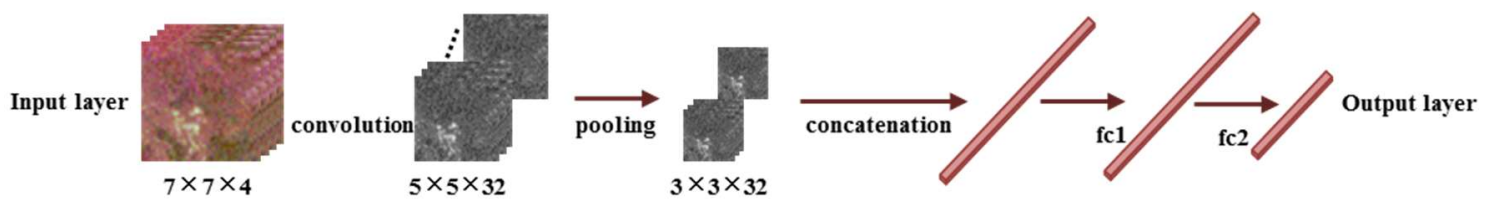

Figure 4. A typical CNN structure. The figure is adapted from Liu et al. [52].

The convolution is formulated as below,

$$
v_{l, t}^{x y}=f\left(b_{l t}+\sum_{k} \sum_{p=0}^{H_{l, t}-1} \sum_{q=0}^{W_{l, t}-1} \omega_{l t k}^{p q} v_{l-1, k}^{(x+p)(y+q)}\right),
$$

where $v_{l, t}^{x y}$ stands for the output at position $(x, y)$ of the $t$ th feature map at the $l$ th layer, $f$ denotes the non-linear activation function, $b_{l t}$ refers to the bias term, and $k$ indexes over the set of feature maps of the (l-1)th layer, $H_{l, t}$ and $W_{l, t}$ are the height and width of $t$ th kernel of $l$ th layer and are indexed by $p$ and $q$, respectively, $\omega_{l t k}^{p q}$ is the convolution kernel value at position $(p, q)$ of the $t$ th feature map in the $l$ th layer connected to the $k$ th feature map in the (l-1)th layer. The recently developed rectified linear units (ReLU) [70] is used as $f$ in this study:

$$
f(x)=\max (0, x) .
$$

For the edge pixels in the input image patch, the convolution is usually conducted by enlarging the input images with zero values (i.e., zero padding). Convolutions with zero padding can keep the input feature map size unchanged and lose little information.

In the $\mathrm{CNN}$ used for object recognition [71], pooling is applied after convolution to derive more abstract and scale invariant features [24], and to reduce noise and avoid overfitting [23]. The pooling can resize the input feature maps spatially. For example, a commonly used max pooling with $2 \times 2$ size and $2 \times 2$ stride will go through the input feature map spatially along both width and height directions and take the maximum value of the $2 \times 2$ features in the input feature map Figure 5(middle). This will only keep a quarter of the input features and discard $75 \%$ of them.

\subsection{CNN Structure Parameter Tuning}

The input patch image size was set to $7 \times 7(K=7)$ as many of the previous studies (Table 1$)$ used such window to balance the computation efficacy and the classification accuracy. Moreover, larger window size may overly smooth the classification results since the classification is implemented on a sliding window basis. The convolution kernel size was set as $3 \times 3$ and the pooling size and stride size were set as $2 \times 2$ following previous studies [16].

Six CNN structures were designed using two different numbers of learnable parameters each with three different pooling strategies. The three different pooling strategies were (Figure 5): (1) no pooling was used and the feature map size was not reduced after convolution, for example, the input $7 \times 7$ image will become a $7 \times 7$ feature map no matter how many layers of $3 \times 3$ convolution were applied (Table 4; Figure 5(left)); (2) max pooling was used and feature map size was reduced after convolution and pooling, for example, the input $7 \times 7$ image will result in a $4 \times 4$ feature map after a $3 \times 3$ convolution and a $2 \times 2$ stride pooling and will result in a $1 \times 1$ feature map after three $3 \times 3$ convolution layers each followed by a $2 \times 2$ stride pooling (Table 4; Figure 5(middle)); and (3) same as (2) but using average pooling (Table 4; Figure 5(right)). Five layer CNN consisting of three convolution layers and two fully connected layers were used in this study. This is because pooling strategies (2) and (3) can have a maximum of three convolution layers (i.e., three convolution layers with $2 \times 2$ stride 
pooling will lead to a $1 \times 1$ feature map) and the convolution layers are usually followed by two fully connected layers.
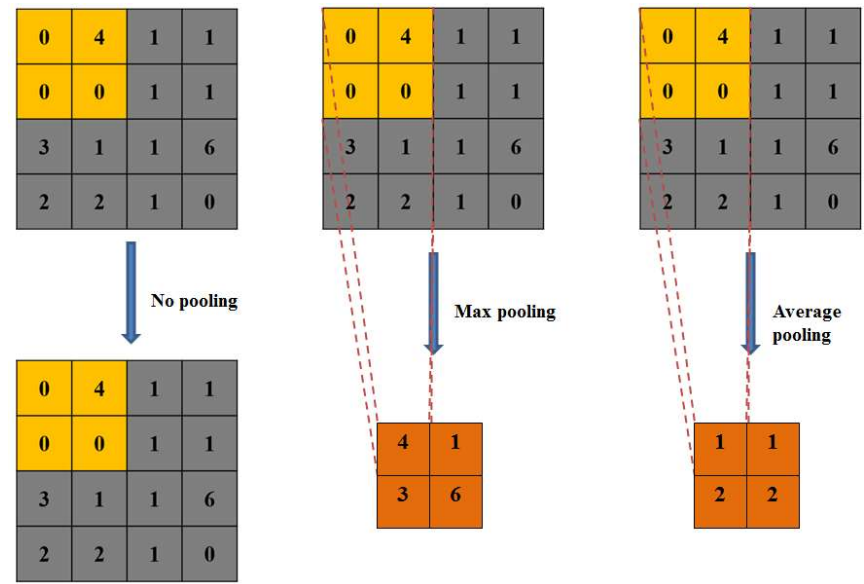

Figure 5. Illustration of no pooling (left), max pooling (middle) and average pooling (right) operations with $2 \times 2$ size and stride.

Two CNN settings with different numbers of learnable parameters $(\sim 70,000$ and $\sim 290,000$ in Table 4) representing different complexity were used to examine the CNN structure complexity effect. Each setting has very similar number of learnable parameters (i.e., similar complexity) so that pooling strategies in each set were fairly compared.

Table 4. Six CNN structures include two settings with significantly different numbers of learnable parameters $(\sim 70,000$ and $\sim 290,000)$ each using three pooling strategies. The number of feature map (in the bracket) is different for $\mathrm{CNN}$ with and without pooling to guarantee that their total numbers of learnable parameters $(n)$ are very similar. Con and FC indicate the convolution and fully connected layer, respectively. The two numbers (with symbol $\times$ ) in each cell outside the bracket indicate the convolution kennel size.

\begin{tabular}{ccccccc}
\hline & \multicolumn{2}{c}{ CNN1 with 70,000 Learnable Parameters } & \multicolumn{2}{c}{ CNN2 with 290,000 Learnable Parameters } \\
\cline { 2 - 6 } & No Pooling & Max Pooling & Avg Pooling & No Pooling & Max Pooling & Avg Pooling \\
\hline$n$ & $79,504 \sim 79,640$ & $77,011 ~ 77,255$ & $77,011 \sim 77,255$ & $296,208 \sim 296,472$ & $290,971 ~ 291,455$ & $290,971 ~ 291,455$ \\
\hline Input & \multicolumn{5}{c}{$7 \times 7 \times 4$ input reflectance } \\
\hline Con layer 1 & $3 \times 3(32)$ & $3 \times 3(59)$ & $3 \times 3(59)$ & $3 \times 3(64)$ & $3 \times 3(119)$ & $3 \times 3(119)$ \\
Con layer 2 & $3 \times 3(32)$ & $3 \times 3(59)$ & $3 \times 3(59)$ & $3 \times 3(64)$ & $3 \times 3(119)$ & $3 \times 3(119)$ \\
Con layer 3 & $3 \times 3(32)$ & $3 \times 3(59)$ & $3 \times 3(59)$ & $3 \times 3(64)$ & $3 \times 3(119)$ & $3 \times 3(119)$ \\
\hline FC layer 1 & $(32)$ & $(64)$ & \\
\hline FC layer 2 & $(8 \sim 12: 11$ for study area 1, 12 for study area 2, 8 for study area 3 and 11 for study 4) \\
\hline \multicolumn{7}{c}{}
\end{tabular}

\subsection{CNN Training Parameterization}

In this study, all the CNNs were trained using the optimal hyperparameters for fair comparison. Following the convention, the input patch reflectance was normalized for each band by being subtracted by mean and divided by standard deviation. The $\mathrm{CNN}$ model was regularized using the weight decay method with coefficient of 0.001 . The mini-batch size was set as 256 . The learning rate started from 0.1 and decreased by 10 times when the validation sample accuracy stopped improving. The validation sample accuracy was calculated and compared every 100 mini-batch gradient descent iterations to check accuracy improvement. In the whole training process, decreasing the learning rate three times can achieve maximum accuracy (i.e., the learning rate changes from $0.1,0.01,0.001$ to 0.0001 ). Following He et al. [72], the validation samples occupied $4 \%$ of the training samples (Table 3 ) for each study area and were randomly selected from them. The start learning rate was different from the recommended 
value of 0.01 in Krizhevsky et al. [16] and He et al. [72] because Ioffe and Szegedy [73] suggested that increasing the start learning rate can get better accuracy. We tested our CNN models and found that the 0.1 start learning rate did give slightly better accuracy without gradient explosion. Such high starting learning rate can be used because the batch normalization regularization technique [73] was adopted to normalize the features generated by each layer. The CNN was implemented using Tensorflow.

\subsection{Classification Result Evaluation}

The random forest classifier [15] was used as a benchmark for comparison. Random forest was run using the same $7 \times 7$ image patch as input. The random forest was implemented using the $\mathrm{R}$ software RANDOMFOREST package (http://www.r-project.org/). The default random forest parameter settings were used (e.g., 500 trees, the number of features used in each split is the squared root of the total number of features, and the number of samples in each tree is $63.2 \%$ of total without replacement).

The accuracy of the generated land cover map was validated both quantitatively and visually. The quantitative indices were only applied to the testing samples shown in Table 3 to avoid the accuracy boosting due to training and testing sample spatial correlation. Four quantitative indices, i.e., the overall accuracy, producer's accuracy, user's accuracy and kappa coefficient were used to evaluate the accuracy of classification of the six CNN structures and the random forest model. In addition, all the study areas were classified, and the land cover classification map was visually assessed by comparing with the true color GaoFen-1 image and the reference land cover map.

Although the imbalanced training samples are on purpose used so that they are proportional to the ground truth class occurrence $[10,68]$, we also compared the random forest and the optimal $\mathrm{CNN}$ structure using the balanced training dataset. This is to avoid that the rare classes that are difficult to classify may bias the random forest and $\mathrm{CNN}$ comparison. For all the classes with more than 4000 samples, 2000 samples were randomly selected as training and 2000 samples (different from training) as testing. The overall accuracy, producer's accuracy, user's accuracy and kappa coefficient of the testing samples were tabulated and compared.

\section{Results}

\subsection{CNN Structure Parameter Tuning}

Figure 6 shows, for each study area, the testing sample classification overall accuracies (left column) and kappa coefficients (right column) for the six $\mathrm{CNN}$ models and random forest. In Figure 6, the blue color is used to indicate which CNN model among the six gets the best performance so that CNN2-Avg always obtains the best scores. This value is directly compared to the performances obtained by random forest algorithm. The rank of three pooling strategies in the order of increasing performance is no pooling, max pooling and average pooling for all the study area experiments and for both CNN settings with significantly different numbers of learnable parameters. The accuracy advantage of the CNN structure with average pooling over the equivalent without pooling is moderate $(0.2-1.5 \%$ higher overall accuracy and 0.01-0.04 higher kappa coefficient). This may be because the input image patch size is much smaller and there is not much image noise that pooling can suppress. Previous studies showed that either max or average pooling may perform best depending on the data feature distribution [23], while results in this study revealed that the GaoFen-1 data feature distribution is more favorable to average pooling strategy. All the CNN overall accuracies are greater than $85 \%$ indicating good classification performance.

For each $\mathrm{CNN}$ setting, the three pooling operations were on purpose designed to have very similar numbers of learnable parameters so that the three pooling operations were fairly compared. For the best pooling operation (i.e., the average pooling), the CNN model with $\sim 290,000$ learnable parameters (CNN2-Avg) performed slightly better than the light model with $\sim 70,000$ learnable parameters (CNN1-Avg). This is reasonable considering that more learnable parameters give more power to $\mathrm{CNN}$ representation. However, the more than four times learnable parameters only give $<0.5 \%$ and $<1 \%$ 
improvement indicating that the $\mathrm{CNN}$ structure used in this study has marginal improvement space without more complicated CNN techniques (e.g., skip connections).
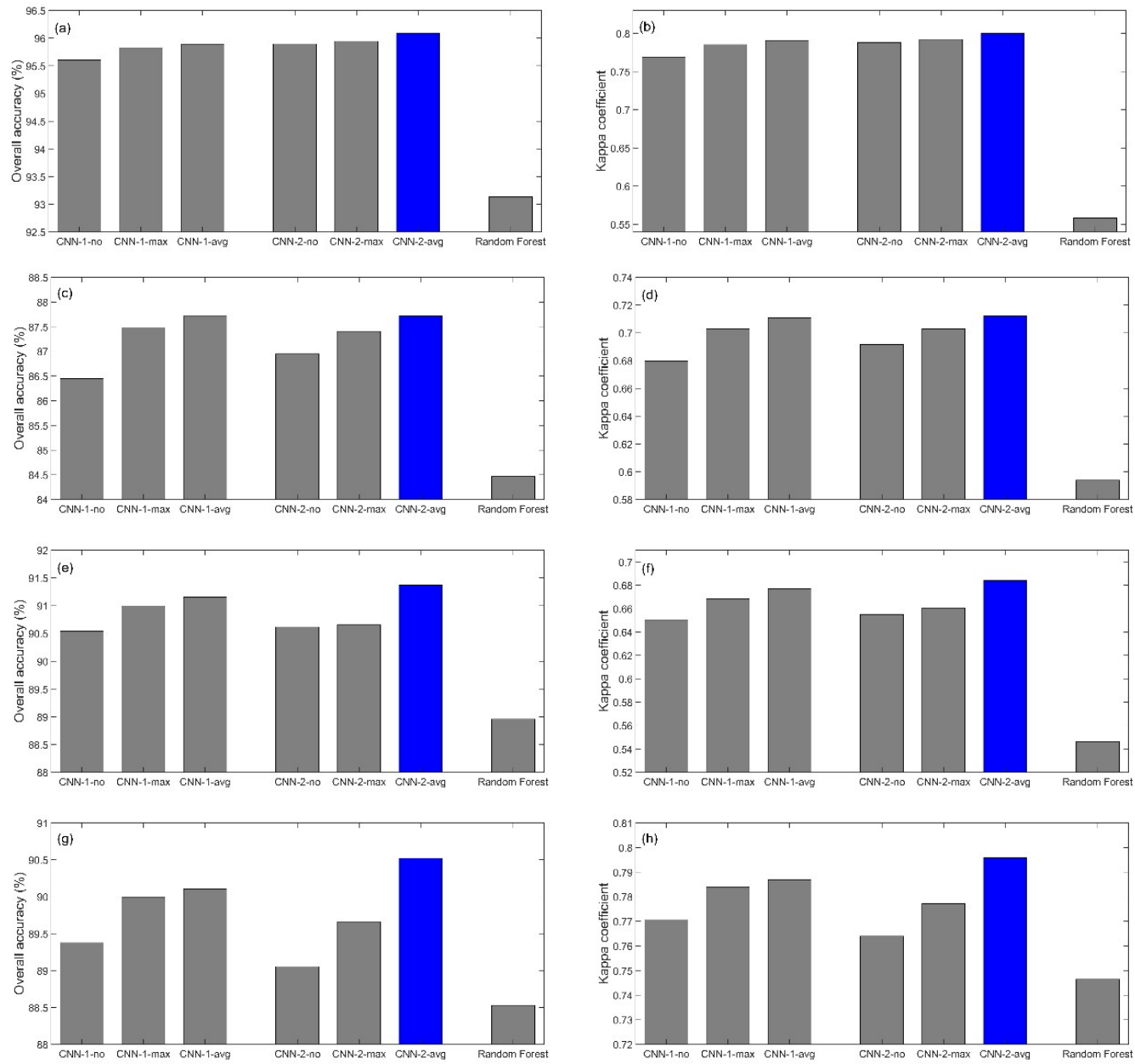

Figure 6. (a) and (b) show the six CNN model and random forest overall accuracies (a) and kappa coefficients (b) of the study area 1 testing sample classification. (c) and (d) show the same for study area 2 testing sample classification, (e) and (f) for study area 3 testing sample classification, and (g) and (h) for study area 4 testing sample classification. CNN1 and CNN2 indicate the first and second $\mathrm{CNN}$ structure settings with $\sim 70,000$ and $\sim 290,000$ learnable parameters, respectively. -no, -max and -avg indicate no, max and average pooling, respectively. The blue color is used to indicate the best performance CNN model among the six models.

\subsection{CNN and Random Forest Classification Accuracy Comparison}

The CNN2-Avg has 2.4-3.3\% higher overall accuracy and 0.05-0.24 higher kappa coefficient (Figure 6) than the random forest using the same $7 \times 7$ input image patch indicting the more powerful capability of $\mathrm{CNN}$ to explore high level spatial structural information. The kappa coefficient improvement of the $\mathrm{CNN}$ model over the random forest model is much larger than the overall accuracy improvement because the user's and producer's accuracies are better balanced in the CNN model classification. Table 5 shows the user's and producer's accuracies for the four study area testing samples. For example, the rare class wheat in Table 5a has more than $60 \%$ user's and producer's accuracy difference for random forest and has only $<13 \%$ difference for $\mathrm{CNN}$ classification. Other such rare class examples include rice, mung bean and bare land in Table $5 a$, wheat, orchards and road in Table $5 b$, maize, vegetable and road in Table $5 \mathrm{c}$, and wheat, soybean and residential in Table $5 \mathrm{~d}$. Some user's accuracy is $\mathrm{NaN}$ (Not a Number, indicating no value) as the total number of pixels classified as such 
class is zero and is used as dividend for user's accuracy calculation. Note that the producer's accuracy cannot be $\mathrm{NaN}$ as there is always some ground truth samples in the testing samples.

Table 5. Study areas 1-4 testing samples (Table 3) accuracies of the random forest and the best CNN model (i.e., the CNN with average pooling and $\sim 290,000$ learnable parameters). The training sample percent to the total training samples (the value is same for the testing sample in Table 3 ) is shown in the bracket after class name.

\begin{tabular}{|c|c|c|c|c|}
\hline \multirow{2}{*}{ Classes } & \multicolumn{2}{|c|}{ Random Forest } & \multicolumn{2}{|c|}{ CNN2-Avg } \\
\hline & User's & Producer's & User's & Producer's \\
\hline \multicolumn{5}{|c|}{ (a) Study Area 1 Testing Sample (Table 3) } \\
\hline Maize $(7.14 \%)$ & 89.30 & 54.84 & 85.76 & 85.57 \\
\hline Rice $(0.06 \%)$ & $\mathrm{NaN}$ & 0.00 & 76.25 & 25.52 \\
\hline Wheat $(0.47 \%)$ & 80.48 & 17.99 & 77.56 & 64.93 \\
\hline Mung bean $(0.36 \%)$ & 82.91 & 6.83 & 64.27 & 44.72 \\
\hline Vegetable $(0.14 \%)$ & $\mathrm{NaN}$ & 0.00 & 41.83 & 11.64 \\
\hline Forest $(0.39 \%)$ & 89.29 & 1.60 & 70.19 & 52.97 \\
\hline Grassland $(0.10 \%)$ & $\mathrm{NaN}$ & 0.00 & 38.10 & 1.93 \\
\hline Wetland $(88.84 \%)$ & 93.38 & 99.57 & 97.77 & 98.98 \\
\hline Residential (0.93\%) & 77.81 & 44.81 & 81.25 & 75.64 \\
\hline $\operatorname{Road}(1.33 \%)$ & 79.18 & 3.68 & 61.35 & 39.39 \\
\hline Bare land $(0.24 \%)$ & 98.94 & 58.46 & 91.12 & 82.46 \\
\hline Overall accuracy & \multicolumn{2}{|c|}{93.13} & \multicolumn{2}{|r|}{96.09} \\
\hline Kappa coefficient & \multicolumn{2}{|c|}{0.5586} & \multicolumn{2}{|c|}{0.8001} \\
\hline \multicolumn{5}{|c|}{ (b) Study Area 2 Testing Sample (Table 3) } \\
\hline Maize $(7.17 \%)$ & 79.55 & 35.15 & 67.99 & 62.77 \\
\hline Rice $(73.90 \%)$ & 87.17 & 97.30 & 92.98 & 95.62 \\
\hline Wheat $(0.39 \%)$ & 75.00 & 1.25 & 44.38 & 30.65 \\
\hline Soybean $(2.38 \%)$ & 82.19 & 67.16 & 80.88 & 78.49 \\
\hline Vegetable $(0.10 \%)$ & $\mathrm{NaN}$ & 0.00 & 46.38 & 16.75 \\
\hline Orchards $(0.63 \%)$ & 95.97 & 10.14 & 72.71 & 55.41 \\
\hline Forest (5.21\%) & 58.76 & 57.76 & 72.32 & 72.76 \\
\hline Grassland $(0.71 \%)$ & $\mathrm{NaN}$ & 0.00 & 18.39 & 8.30 \\
\hline Water $(5.90 \%)$ & 76.92 & 64.40 & 77.90 & 74.71 \\
\hline Residential (1.71\%) & 62.15 & 61.67 & 69.37 & 65.35 \\
\hline Road (1.47\%) & 71.92 & 27.82 & 62.53 & 51.22 \\
\hline Bare land $(0.43 \%)$ & 100.00 & 0.13 & 28.57 & 17.00 \\
\hline Overall accuracy & \multicolumn{2}{|c|}{84.47} & \multicolumn{2}{|r|}{87.72} \\
\hline Kappa coefficient & \multicolumn{2}{|c|}{0.5939} & \multicolumn{2}{|c|}{0.7121} \\
\hline \multicolumn{5}{|c|}{ (c) Study Area 3 Testing Sample (Table 3) } \\
\hline Maize (2.84\%) & 89.71 & 4.74 & 76.84 & 34.80 \\
\hline Rice $(83.26 \%)$ & 91.55 & 99.38 & 94.59 & 98.62 \\
\hline Vegetable $(1.66 \%)$ & 66.40 & 10.90 & 58.62 & 48.57 \\
\hline Forest $(2.42 \%)$ & 52.25 & 20.67 & 61.00 & 53.01 \\
\hline Grassland (1.16\%) & 25.00 & 0.10 & 34.41 & 13.25 \\
\hline Water $(2.29 \%)$ & 69.70 & 58.91 & 73.03 & 67.42 \\
\hline Residential (4.79\%) & 64.94 & 80.13 & 79.15 & 77.90 \\
\hline Road (1.58\%) & 89.01 & 22.02 & 65.21 & 47.67 \\
\hline Overall accuracy & \multicolumn{2}{|c|}{88.96} & \multicolumn{2}{|r|}{91.37} \\
\hline Kappa coefficient & \multicolumn{2}{|c|}{0.5463} & \multicolumn{2}{|c|}{0.6842} \\
\hline \multicolumn{5}{|c|}{ (d) Study Area 4 Testing Sample (Table 3) } \\
\hline Maize $(71.76 \%)$ & 94.11 & 97.73 & 96.03 & 97.38 \\
\hline Rice $(7.73 \%)$ & 89.36 & 87.82 & 90.96 & 92.90 \\
\hline Wheat $(0.20 \%)$ & 87.10 & 38.85 & 70.63 & 81.30 \\
\hline Soybean $(0.59 \%)$ & 88.40 & 63.02 & 92.57 & 84.91 \\
\hline Vegetable $(0.54 \%)$ & $\mathrm{NaN}$ & 0.00 & 17.98 & 8.40 \\
\hline Forest $(4.70 \%)$ & 51.57 & 64.68 & 68.56 & 68.12 \\
\hline Grassland $(0.99 \%)$ & 86.36 & 5.48 & 35.18 & 21.07 \\
\hline Water $(4.18 \%)$ & 94.89 & 80.86 & 93.95 & 92.15 \\
\hline Residential (3.04\%) & 62.91 & 87.32 & 70.98 & 75.53 \\
\hline Road (4.77\%) & 52.20 & 33.86 & 61.69 & 56.32 \\
\hline Bare land $(1.49 \%)$ & 38.01 & 13.58 & 37.30 & 33.82 \\
\hline Overall accuracy & & .53 & & 90.52 \\
\hline Kappa coefficient & & 464 & & 0.7959 \\
\hline
\end{tabular}




\subsection{CNN and Random Forest Land Cover Map Comparison}

Figures 7-10 show the $8 \mathrm{~m}$ GaoFen-1 true color image, $2 \mathrm{~m}$ pan-sharpened image, reference land cover map, two land cover maps produced by the best CNN structure (CNN2 with average pooling) and the random forest models, for the four $400 \times 4002 \mathrm{~m}$ pixel example areas shown in Figure 3. The pan-sharpened images have more spatial details which are favorable for small crop field classification. The random forest classification maps are inferior to (i.e., less resembling to the reference land cover maps than) the CNN classification maps. This is because the end-to-end CNN training process can extract spatial information which is helpful to classify the spectrally similar classes, such as the maize and grassland classes in Figure 7, rice and wheat classes in Figure 8, rice and maize classes in Figure 9, and road and bare land classes in Figure 10. Due to the same reason, the linear features (e.g., roads and roadside forests in Figures 7,9 and 10) are more evident in the CNN classification maps.

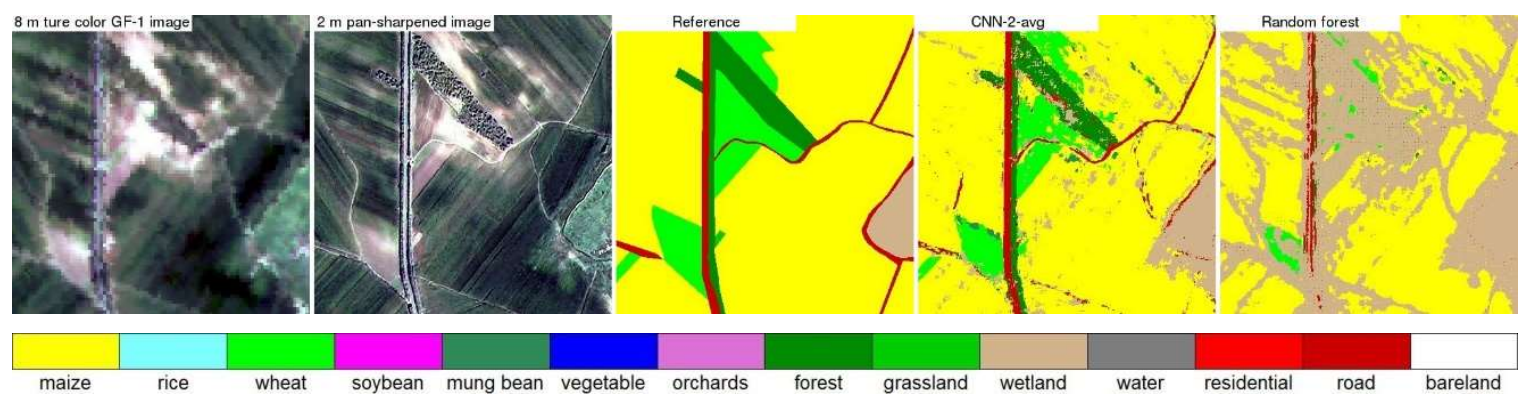

Figure 7. Study area 1 example $8 \mathrm{~m}$ GaoFen- 1 true color image (the $400 \times 4002 \mathrm{~m}$ pixel box in Figure 3), $2 \mathrm{~m}$ pan-sharpened image, and three $2 \mathrm{~m}$ land cover maps (the reference land cover map, the land cover map generated by CNN model with average pooling and $\sim 290,000$ learnable parameters, and the land cover map generated by the random forest model). The two true color images are displayed using the same stretch.

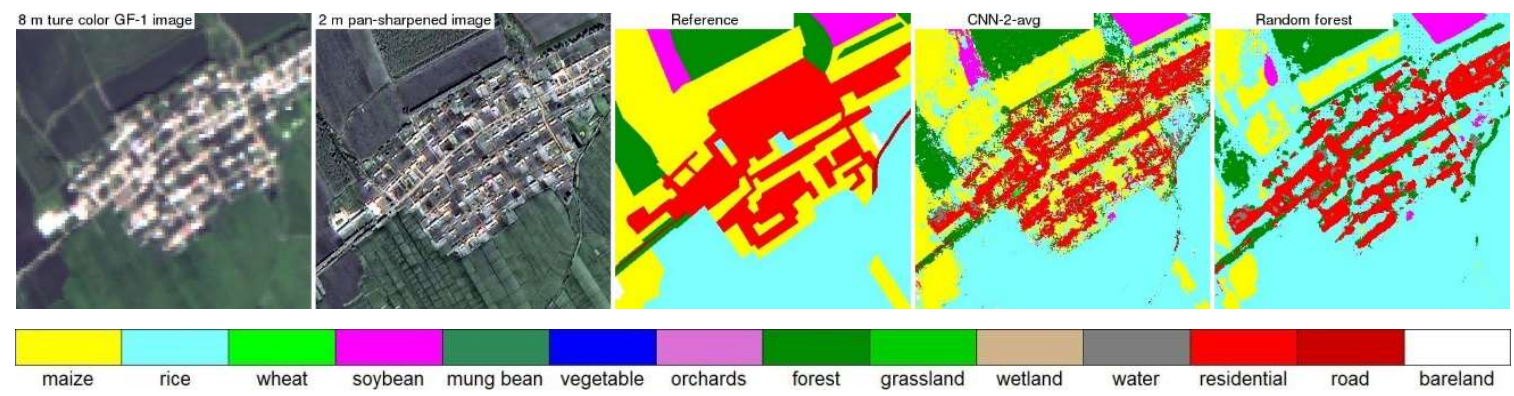

Figure 8. Same as Figure 7 but for the example $400 \times 400$ pixels in the box area in study area 2 (Figure 3).

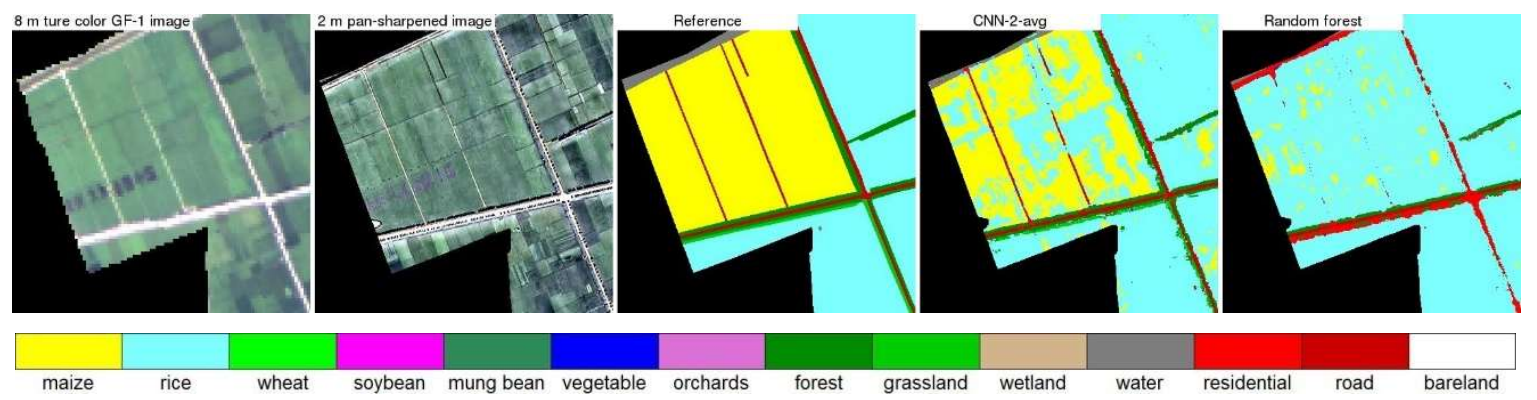

Figure 9. Same as Figure 7 but for the example $400 \times 400$ pixels in the box area in study area 3 (Figure 3). 


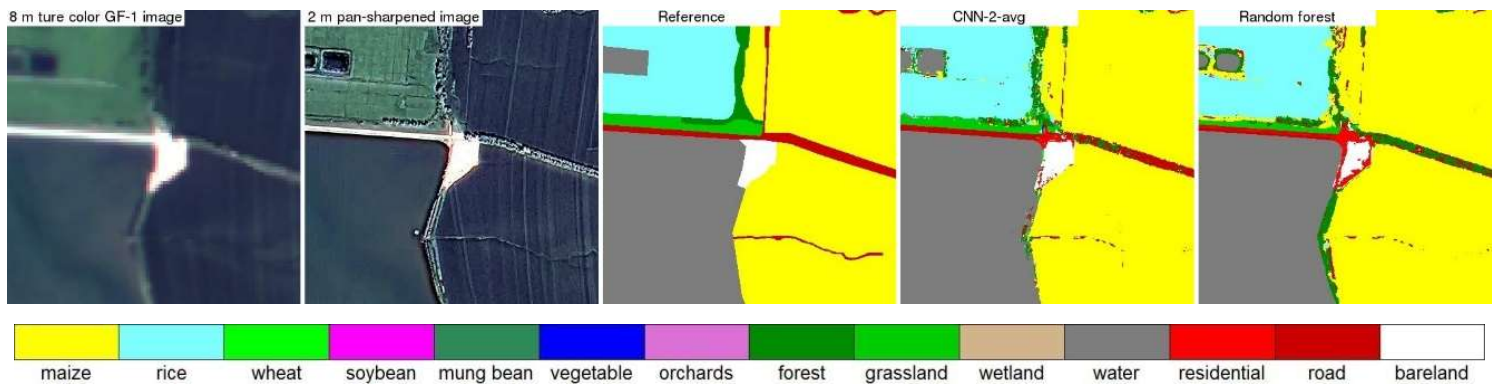

Figure 10. Same as Figure 7 but for the example $400 \times 400$ pixels in the box area in study area 4 (Figure 3).

\subsection{CNN and Random Forest Accuracy Comparison for Balanced Training Data}

All the previous results were derived from the training samples which are proportional to the land cover occurrence in the reference land cover maps. To examine the training sample balance effect, in this section, the CNN and random forest were trained using the sample data balanced among different classes, i.e., same number of training samples for all the classes. Table 6 lists the balanced testing dataset classification accuracies for the four study areas. The random forest is still inferior to the $\mathrm{CNN}$ model by 3.8-9.1\% overall accuracy and 0.04-0.10 kappa coefficients. However, the overall accuracy of $\mathrm{CNN}$ model is only $78-85 \%$ that is lower than the imbalanced training dataset (Table 5). This is because overall accuracy may be boosted for imbalanced dataset by simply classifying most pixels as the majority classes. Similarly, the user's and producer's accuracies for CNN are more balanced than that for random forest.

Table 6. Balanced sample accuracies of the random forest and the CNN2-Avg model for study areas 1-4 samples data. A total of 2000 training and 2000 testing samples were randomly selected from Table 3 (class with samples $<4000$ was not used). The training sample percent to the total training samples (the value is same for the testing sample) is shown in the bracket after class name.

\begin{tabular}{|c|c|c|c|c|}
\hline \multirow{2}{*}{ Classes } & \multicolumn{2}{|c|}{ Random Forest } & \multicolumn{2}{|c|}{ CNN2-Avg } \\
\hline & User's & Producer's & User's & Producer's \\
\hline \multicolumn{5}{|c|}{ (a) Study Area 1 Testing Sample } \\
\hline Maize (25.00\%) & 84.38 & 82.95 & 85.62 & 88.40 \\
\hline Wetland $(25.00 \%)$ & 76.74 & 72.60 & 81.88 & 79.30 \\
\hline Residential (25.00\%) & 83.21 & 86.00 & 89.66 & 87.10 \\
\hline Road $(25.00 \%)$ & 64.92 & 67.35 & 72.80 & 74.80 \\
\hline Overall accuracy & \multicolumn{2}{|c|}{77.26} & \multicolumn{2}{|c|}{82.40} \\
\hline Kappa coefficient & \multicolumn{2}{|c|}{0.6963} & \multicolumn{2}{|c|}{0.7653} \\
\hline \multicolumn{5}{|c|}{ (b) Study Area 2 Testing Sample } \\
\hline Maize (14.29\%) & 74.92 & 57.35 & 71.18 & 65.95 \\
\hline Rice (14.29\%) & 69.43 & 80.05 & 74.50 & 79.45 \\
\hline Soybean (14.29\%) & 83.25 & 89.20 & 88.73 & 90.90 \\
\hline Forest (14.29\%) & 60.66 & 70.85 & 73.16 & 73.85 \\
\hline Water $(14.29 \%)$ & 82.09 & 62.35 & 73.89 & 74.15 \\
\hline Residential (14.29\%) & 75.58 & 79.70 & 85.44 & 85.10 \\
\hline $\operatorname{Road}(14.29 \%)$ & 63.23 & 65.00 & 77.25 & 75.20 \\
\hline Overall accuracy & \multicolumn{2}{|c|}{70.73} & \multicolumn{2}{|c|}{77.80} \\
\hline Kappa coefficient & \multicolumn{2}{|c|}{0.6742} & \multicolumn{2}{|c|}{0.7410} \\
\hline
\end{tabular}


Table 6. Cont.

\begin{tabular}{|c|c|c|c|c|}
\hline \multirow{2}{*}{ Classes } & \multicolumn{2}{|c|}{ Random Forest } & \multicolumn{2}{|c|}{ CNN2-Avg } \\
\hline & User's & Producer's & User's & Producer's \\
\hline \multicolumn{5}{|c|}{ (c) Study Area 3 Testing Sample } \\
\hline Maize $(20.00 \%)$ & 76.43 & 67.95 & 80.57 & 79.00 \\
\hline Rice $(20.00 \%)$ & 65.60 & 72.75 & 73.49 & 76.50 \\
\hline Forest $(20.00 \%)$ & 58.58 & 67.45 & 73.27 & 75.10 \\
\hline Water $(20.00 \%)$ & 82.36 & 69.80 & 83.14 & 80.60 \\
\hline Residential (20.00\%) & 78.61 & 78.85 & 86.28 & 84.90 \\
\hline Overall accuracy & \multicolumn{2}{|c|}{70.08} & \multicolumn{2}{|c|}{79.22} \\
\hline Kappa coefficient & \multicolumn{2}{|c|}{0.6420} & \multicolumn{2}{|c|}{0.7403} \\
\hline \multicolumn{5}{|c|}{ (d) Study Area 4 Testing Sample } \\
\hline Maize $(16.67 \%)$ & 92.19 & 86.70 & 89.74 & 89.70 \\
\hline Rice $(16.67 \%)$ & 93.00 & 89.65 & 91.78 & 92.70 \\
\hline Forest $(16.67 \%)$ & 61.89 & 74.80 & 74.81 & 72.75 \\
\hline Water $(16.67 \%)$ & 96.06 & 81.70 & 93.47 & 91.60 \\
\hline Residential (16.67\%) & 83.88 & 92.65 & 86.85 & 89.50 \\
\hline $\operatorname{Road}(16.67 \%)$ & 66.47 & 61.95 & 72.85 & 73.40 \\
\hline Overall accuracy & \multicolumn{2}{|c|}{81.15} & \multicolumn{2}{|c|}{84.94} \\
\hline Kappa coefficient & \multicolumn{2}{|c|}{0.7749} & \multicolumn{2}{|c|}{0.8193} \\
\hline
\end{tabular}

\section{Discussion}

CNN has been used for land cover classifications using remote sensing data, in particular hyperspectral data $[42,43,48,49,64]$. In this study, CNN was evaluated for mapping smallholder agriculture considering the unique spatial pattern of the smallholder agricultural landscapes. The CNN structures with three different pooling strategies were carefully designed with very similar numbers of learnable parameters and they were trained using the same optimal hyperparameters. The classification results showed that the pooling is still necessary even for small input image patch (i.e., $7 \times 7$ input image patch) because the small patch size input image still contains a moderate level of irrelevant information that the pooling can suppress. The average pooling performed better than max pooling because the max pooling is more suitable for separation of features that are very sparse [23] which is not the case for a small $7 \times 7$ input image patch.

In this study, the simple Gram-Schmidt method was used to pan-sharpen the $8 \mathrm{~m}$ multispectral images. Its adoption can be considered a reasonable trade-off between performance and easy-to-use. The Gram-Schmidt method, in fact, has performance that can be considered acceptable, even though it is not an up-to-date method and other pansharpening methods produce better scores [74-77]. Anyway, its adoption presents undeniable advantages in terms of easy-to-use because Gram-Schmidt is included in commercial software.

The CNN classifications have better accuracy than the random forest classifications as the CNN can explore the high level spatial information. However, there is still some pepper and salt effect in the CNN land cover classification maps. They occur mostly in the heterogeneous area, e.g., the residential and maize mixed area in Figure 8 and the roads and roadside forests mixed area in Figure 10. This is because in such heterogeneous area with two land cover classes distributed in a checkerboard pattern, all pixels may have very similar spatial context whatever the pixel class is. To handle such issue, Zhang et al. [56] and Pan and Zhao [78] have tried to fuse CNN and Markov based classification results at the decision level. Other options to handle this may use multi-scale CNN [44,79], object based CNN $[80,81]$ and fully convolutional network $[82,83]$.

The CNN had better performance than random forest for both imbalanced and balanced training datasets. In the imbalanced dataset, the classes with rare samples are difficult to classify and random forest easily misclassified these classes. The CNN performed much better for classes with rare samples and has a very high kappa coefficient improvement compared to the random forest. Furthermore, 
in this study the CNN loss function is defined as the average loss function of all the training samples. Training sample loss function can be easily weighted based on their classes to better classify specific classes. This may be useful when user's accuracy is more or less important than the producer's accuracy, for example, in cloud detection, omission error has a more severe effect on time series application than commission error [84].

\section{Conclusions}

This study evaluated CNN for remote sensing image classification of smallholder agricultural landscape using pan-sharpened GaoFen-1 satellite data with $2 \mathrm{~m}$ spatial resolution. Four study areas in Heilongjiang province were selected with reference land cover maps generated by manual interpretation. Pixels were systematically sampled from the reference land cover maps and evenly split for training and testing. Six CNN structures were designed and divided into two settings based on their number of learnable parameters (i.e., a 70,000 and a $\sim 290,000$ learnable parameter setting). Each setting has three different pooling strategies, i.e., without pooling, with max pooling and with average pooling. Results showed that $\mathrm{CNN}$ performed better than the established random forest classifier. This is because the CNN end-to-end training can effectively extract spatial information favorable to discriminate spectrally similar classes which occurs frequently in smallholder agricultural landscapes and especially in the four-band multispectral images. Random forest, despite taking the same $7 \times 7$ image patch as input, can only consider each pixel separately during the tree split training process.

Author Contributions: Conceptualization H.K.Z. and B.X.; Implementation B.X. and H.K.Z.; Writing and Editing H.K.Z., B.X., and J.X.

Funding: This research was funded by the National Natural Science Foundation of China (41401517) and the Zhejiang Basic Public Welfare Research Project (LGF19D010002).

Conflicts of Interest: The authors declare no conflict of interest.

\section{References}

1. Hansen, M.C.; Loveland, T.R. A review of large area monitoring of land cover change using Landsat data. Remote Sens. Environ. 2012, 122, 66-74. [CrossRef]

2. Gómez, C.; White, J.C.; Wulder, M.A. Optical remotely sensed time series data for land cover classification: A review. ISPRS J. Photogramm. Remote Sens. 2016, 116, 55-72. [CrossRef]

3. Phiri, D.; Morgenroth, J. Developments in Landsat land cover classification methods: A review. Remote Sens. 2017, 9, 967. [CrossRef]

4. Wulder, M.A.; Coops, N.C.; Roy, D.P.; White, J.C.; Hermosilla, T. Land cover 2.0. Int. J. Remote Sens. 2018, 39, 4254-4284. [CrossRef]

5. Bauer, M.E.; Cipra, J.E.; Anuta, P.E.; Etheridge, J.B. Identification and area estimation of agricultural crops by computer classification of Landsat MSS data. Remote Sens. Environ. 1979, 8, 77-92. [CrossRef]

6. Hansen, M.C.; Potapov, P.V.; Moore, R.; Hancher, M.; Turubanova, S.A.; Tyukavina, A.; Kommareddy, A.; Thau, D.; Stehman, S.V.; Goetz, S.J.; et al. High-resolution global maps of 21st-century forest cover change. Science 2013, 342, 850-853. [CrossRef] [PubMed]

7. Hermosilla, T.; Wulder, M.A.; White, J.C.; Coops, N.C.; Hobart, G.W. Disturbance-Informed Annual Land Cover Classification Maps of Canada's Forested Ecosystems for a 29-Year Landsat Time Series. Can. J. Remote Sens. 2018, 44, 67-87. [CrossRef]

8. Gong, P.; Wang, J.; Yu, L.; Zhao, Y.; Zhao, Y.; Liang, L.; Niu, Z.; Huang, X.; Fu, H.; Liu, S.; et al. Finer resolution observation and monitoring of global land cover: First mapping results with Landsat TM and ETM+ data. Int. J. Remote Sens. 2013, 34, 2607-2654. [CrossRef]

9. Chen, J.; Chen, J.; Liao, A.; Cao, X.; Chen, L.; Chen, X.; He, C.; Han, G.; Peng, S.; Lu, M. Global land cover mapping at $30 \mathrm{~m}$ resolution: A POK-based operational approach. ISPRS J. Photogramm. Remote Sens. 2015, 103, 7-27. [CrossRef] 
10. Zhang, H.K.; Roy, D.P. Using the $500 \mathrm{~m}$ MODIS land cover product to derive a consistent continental scale $30 \mathrm{~m}$ Landsat land cover classification. Remote Sens. Environ. 2017, 197, 15-34. [CrossRef]

11. Zhu, Z.; Gallant, A.L.; Woodcock, C.E.; Pengra, B.; Olofsson, P.; Loveland, T.R.; Jin, S.; Dahal, D.; Yang, L.; Auch, R.F. Optimizing selection of training and auxiliary data for operational land cover classification for the LCMAP initiative. ISPRS J. Photogramm. Remote Sens. 2016, 122, 206-221. [CrossRef]

12. Wulder, M.A.; Masek, J.G.; Cohen, W.B.; Loveland, T.R.; Woodcock, C.E. Opening the archive: How free data has enabled the science and monitoring promise of Landsat. Remote Sens. Environ. 2012, 122, 2-10. [CrossRef]

13. Dwyer, J.L.; Roy, D.P.; Sauer, B.; Jenkerson, C.B.; Zhang, H.K.; Lymburner, L. Analysis Ready Data: Enabling analysis of the Landsat archive. Remote Sens. 2018, 10, 1363.

14. Vapnik, V.N. The Nature of Statistical Learning Theory; Springer: New York, NY, USA, 1995.

15. Breiman, L. Random forests. Mach. Learn. 2001, 45, 5-32. [CrossRef]

16. Krizhevsky, A.; Sutskever, I.; Hinton, G.E. Imagenet classification with deep convolutional neural networks. In Proceedings of the 26th Annual Conference on Neural Information Processing Systems, Lake Tahoe, NV, USA, 3-6 December 2012; pp. 1097-1105.

17. Chen, Y.; Lin, Z.; Zhao, X.; Wang, G.; Gu, Y. Deep learning-based classification of hyperspectral data. IEEE J. Sel. Top. Appl. Earth Obs. Remote Sens. 2014, 7, 2094-2107. [CrossRef]

18. Lyu, H.; Lu, H.; Mou, L.; Li, W.; Wright, J.; Li, X.; Li, X.; Zhu, X.X.; Wang, J.; Yu, L.; et al. Long-term annual mapping of four cities on different continents by applying a deep information learning method to landsat data. Remote Sens. 2018, 10, 471. [CrossRef]

19. Basaeed, E.; Bhaskar, H.; Hill, P.; Al-Mualla, M.; Bull, D. A supervised hierarchical segmentation of remote-sensing images using a committee of multi-scale convolutional neural networks. Int. J. Remote Sens. 2016, 37, 1671-1691. [CrossRef]

20. Xia, M.; Liu, W.A.; Shi, B.; Weng, L.; Liu, J. Cloud/snow recognition for multispectral satellite imagery based on a multidimensional deep residual network. Int. J. Remote Sens. 2019, 40, 156-170. [CrossRef]

21. LeCun, Y.; Bengio, Y.; Hinton, G. Deep learning. Nature 2015, 521, 436. [CrossRef]

22. Hinton, G.E.; Osindero, S.; Teh, Y.-W. A fast learning algorithm for deep belief nets. Neural Comput. 2006, 18, 1527-1554. [CrossRef]

23. Boureau, Y.L.; Ponce, J.; LeCun, Y. A theoretical analysis of feature pooling in visual recognition. In Proceedings of the 27th International Conference on Machine Learning, Haifa, Israel, 21-24 June 2010; pp. 111-118.

24. Scherer, D.; Müller, A.; Behnke, S. Evaluation of pooling operations in convolutional architectures for object recognition. In Proceedings of the 20th International Conference on Artificial Neural Networks, Thessaloniki, Greece, 15-18 September 2010; pp. 92-101.

25. Christiansen, P.; Nielsen, L.; Steen, K.; Jørgensen, R.; Karstoft, H. DeepAnomaly: Combining background subtraction and deep learning for detecting obstacles and anomalies in an agricultural field. Sensors 2016, 16, 1904. [CrossRef] [PubMed]

26. dos Santos Ferreira, A.; Freitas, D.M.; da Silva, G.G.; Pistori, H.; Folhes, M.T. Weed detection in soybean crops using ConvNets. Comput. Electron. Agric. 2017, 143, 314-324. [CrossRef]

27. Zheng, Y.Y.; Kong, J.L.; Jin, X.B.; Wang, X.Y.; Zuo, M. CropDeep: The Crop Vision Dataset for Deep-Learning-Based Classification and Detection in Precision Agriculture. Sensors 2019, 19, 1058. [CrossRef] [PubMed]

28. Kamilaris, A.; Prenafeta-Boldú, F.X. Deep learning in agriculture: A survey. Comput. Electron. Agric. 2018, 147, 70-90. [CrossRef]

29. Kussul, N.; Lavreniuk, M.; Skakun, S.; Shelestov, A. Deep learning classification of land cover and crop types using remote sensing data. IEEE Geosci. Remote Sens. Lett. 2017, 14, 778-782. [CrossRef]

30. Zhong, L.; Hu, L.; Zhou, H. Deep learning based multi-temporal crop classification. Remote Sens. Environ. 2019, 221, 430-443. [CrossRef]

31. Ji, S.; Zhang, C.; Xu, A.; Shi, Y.; Duan, Y. 3D Convolutional Neural Networks for Crop Classification with Multi-Temporal Remote Sensing Images. Remote Sens. 2018, 10, 75. [CrossRef]

32. Sidike, P.; Sagan, V.; Maimaitijiang, M.; Maimaitiyiming, M.; Shakoor, N.; Burken, J.; Mockler, T.; Fritschi, F.B. dPEN: Deep Progressively Expanded Network for mapping heterogeneous agricultural landscape using WorldView-3 satellite imagery. Remote Sens. Environ. 2019, 221, 756-772. [CrossRef] 
33. Yu, L.; Wang, J.; Clinton, N.; Xin, Q.; Zhong, L.; Chen, Y.; Gong, P. FROM-GC: 30 m global cropland extent derived through multisource data integration. Int. J. Digit. Earth 2013, 6, 521-533. [CrossRef]

34. Yu, L.; Wang, J.; Li, X.; Li, C.; Zhao, Y.; Gong, P. A multi-resolution global land cover dataset through multisource data aggregation. Sci. China Earth Sci. 2014, 57, 2317-2329. [CrossRef]

35. Xiong, J.; Thenkabail, P.S.; Tilton, J.C.; Gumma, M.K.; Teluguntla, P.; Oliphant, A.; Congalton, R.G.; Yadav, K.; Gorelick, N. Nominal 30-m cropland extent map of continental Africa by integrating pixel-based and object-based algorithms using Sentinel-2 and Landsat-8 data on Google Earth Engine. Remote Sens. 2017, 9, 1065. [CrossRef]

36. Samberg, L.H.; Gerber, J.S.; Ramankutty, N.; Herrero, M.; West, P.C. Subnational distribution of average farm size and smallholder contributions to global food production. Environ. Res. Lett. 2016, 11, 124010. [CrossRef]

37. Aguilar, R.; Zurita-Milla, R.; Izquierdo-Verdiguier, E.; A De By, R. A cloud-based multi-temporal ensemble classifier to map smallholder farming systems. Remote Sens. 2018, 10, 729. [CrossRef]

38. Neigh, C.S.R.; Carroll, M.L.; Wooten, M.R.; McCarty, J.L.; Powell, B.F.; Husak, G.J.; Enenkel, M.; Hain, C.R. Smallholder crop area mapped with wall-to-wall WorldView sub-meter panchromatic image texture: A test case for Tigray, Ethiopia. Remote Sens. Environ. 2018, 212, 8-20. [CrossRef]

39. Fritz, S.; See, L.; McCallum, I.; You, L.; Bun, A.; Moltchanova, E.; Duerauer, M.; Albrecht, F.; Schill, C.; Perger, C.; et al. Mapping global cropland and field size. Glob. Chang. Biol. 2015, 21, 1980-1992. [CrossRef]

40. Zhang, W.; Cao, G.; Li, X.; Zhang, H.; Wang, C.; Liu, Q.; Chen, X.; Cui, Z.; Shen, J.; Jiang, R.; et al. Closing yield gaps in China by empowering smallholder farmers. Nature 2016, 537, 671-674. [CrossRef]

41. Simonyan, K.; Zisserman, A. Very Deep Convolutional Networks for Large-Scale Image Recognition. arXiv 2014, arXiv:1409.1556.

42. Chen, Y.; Jiang, H.; Li, C.; Jia, X.; Ghamisi, P. Deep feature extraction and classification of hyperspectral images based on convolutional neural networks. IEEE Trans. Geosci. Remote Sens. 2016, 54, 6232-6251. [CrossRef]

43. Zhao, W.; Du, S. Spectral-spatial feature extraction for hyperspectral image classification: A dimension reduction and deep learning approach. IEEE Trans. Geosci. Remote Sens. 2016, 54, 4544-4554. [CrossRef]

44. Guo, Z.; Shao, X.; Xu, Y.; Miyazaki, H.; Ohira, W.; Shibasaki, R. Identification of village building via google earth images and supervised machine learning methods. Remote Sens. 2016, 8, 271. [CrossRef]

45. Li, Y.; Zhang, H.; Shen, Q. Spectral-spatial classification of hyperspectral imagery with 3D convolutional neural network. Remote Sens. 2017, 9, 67. [CrossRef]

46. Mei, S.; Ji, J.; Hou, J.; Li, X.; Du, Q. Learning sensor-specific spatial-spectral features of hyperspectral images via convolutional neural networks. IEEE Trans. Geosci. Remote Sens. 2017, 55, 4520-4533. [CrossRef]

47. Santara, A.; Mani, K.; Hatwar, P.; Singh, A.; Garg, A.; Padia, K.; Mitra, P. BASS Net: Band-adaptive spectral-spatial feature learning neural network for hyperspectral image classification. IEEE Trans. Geosci. Remote Sens. 2017, 55, 5293-5301. [CrossRef]

48. Yang, J.; Zhao, Y.Q.; Chan, J.C.W. Learning and transferring deep joint spectral-spatial features for hyperspectral classification. IEEE Trans. Geosci. Remote Sens. 2017, 55, 4729-4742. [CrossRef]

49. Wu, H.; Prasad, S. Convolutional recurrent neural networks forhyperspectral data classification. Remote Sens. 2017, 9, 298. [CrossRef]

50. Hamida, A.B.; Benoit, A.; Lambert, P.; Amar, C.B. 3-D Deep Learning Approach for Remote Sensing Image Classification. IEEE Trans. Geosci. Remote Sens. 2018, 56, 4420-4434. [CrossRef]

51. Xu, X.; Li, W.; Ran, Q.; Du, Q.; Gao, L.; Zhang, B. Multisource remote sensing data classification based on convolutional neural network. IEEE Trans. Geosci. Remote Sens. 2018, 56, 937-949. [CrossRef]

52. Liu, B.; Yu, X.; Zhang, P.; Yu, A.; Fu, Q.; Wei, X. Supervised deep feature extraction for hyperspectral image classification. IEEE Trans. Geosci. Remote Sens. 2018, 56, 1909-1921. [CrossRef]

53. Song, W.; Li, S.; Fang, L.; Lu, T. Hyperspectral image classification with deep feature fusion network. IEEE Trans. Geosci. Remote Sens. 2018, 56, 3173-3184. [CrossRef]

54. Hao, S.; Wang, W.; Ye, Y.; Li, E.; Bruzzone, L. A Deep Network Architecture for Super-Resolution-Aided Hyperspectral Image Classification with Classwise Loss. IEEE Trans. Geosci. Remote Sens. 2018, 56, 4650-4663. [CrossRef]

55. Zhong, Z.; Li, J.; Luo, Z.; Chapman, M. Spectral-Spatial Residual Network for Hyperspectral Image Classification: A 3-D Deep Learning Framework. IEEE Trans. Geosci. Remote Sens. 2018, 56, 847-858. [CrossRef] 
56. Zhang, C.; Sargent, I.; Pan, X.; Gardiner, A.; Hare, J.; Atkinson, P.M. VPRS-based regional decision fusion of CNN and MRF classifications for very fine resolution remotely sensed images. IEEE Trans. Geosci. Remote Sens. 2018, 56, 4507-4521. [CrossRef]

57. Yang, X.; Ye, Y.; Li, X.; Lau, R.Y.; Zhang, X.; Huang, X. Hyperspectral Image Classification with Deep Learning Models. IEEE Trans. Geosci. Remote Sens. 2018, 56, 5408-5423. [CrossRef]

58. Mahdianpari, M.; Salehi, B.; Rezaee, M.; Mohammadimanesh, F.; Zhang, Y. Very deep convolutional neural networks for complex land cover mapping using multispectral remote sensing imagery. Remote Sens. 2018, 10, 1119. [CrossRef]

59. Gao, Q.; Lim, S.; Jia, X. Hyperspectral Image Classification Using Convolutional Neural Networks and Multiple Feature Learning. Remote Sens. 2018, 10, 299. [CrossRef]

60. Karakizi, C.; Karantzalos, K.; Vakalopoulou, M.; Antoniou, G. Detailed Land Cover Mapping from Multitemporal Landsat-8 Data of Different Cloud Cover. Remote Sens. 2018, 10, 1214. [CrossRef]

61. Wei, W.; Zhang, J.; Zhang, L.; Tian, C.; Zhang, Y. Deep Cube-Pair Network for Hyperspectral Imagery Classification. Remote Sens. 2018, 10, 783. [CrossRef]

62. Paoletti, M.; Haut, J.; Plaza, J.; Plaza, A. Deep\&Dense Convolutional Neural Network for Hyperspectral Image Classification. Remote Sens. 2018, 10, 1454.

63. Wang, W.; Dou, S.; Jiang, Z.; Sun, L. A Fast Dense Spectral-Spatial Convolution Network Framework for Hyperspectral Images Classification. Remote Sens. 2018, 10, 1068. [CrossRef]

64. Li, C.; Yang, S.X.; Yang, Y.; Gao, H.; Zhao, J.; Qu, X.; Wang, Y.; Yao, D.; Gao, J. Hyperspectral remote sensing image classification based on maximum overlap pooling convolutional neural network. Sensors 2018, $18,3587$. [CrossRef]

65. Zhang, H.K.; Huang, B. A new look at image fusion methods from a Bayesian perspective. Remote Sens. 2015, 7, 6828-6861. [CrossRef]

66. Zhang, H.K.; Roy, D.P. Computationally inexpensive Landsat 8 Operational Land Imager (OLI) pansharpening. Remote Sens. 2016, 8, 180. [CrossRef]

67. Yu, L.; Wang, J.; Gong, P. Improving $30 \mathrm{~m}$ global land-cover map FROM-GLC with time series MODIS and auxiliary data sets: A segmentation-based approach. Int. J. Remote Sens. 2013, 34, 5851-5867. [CrossRef]

68. Colditz, R.R. An evaluation of different training sample allocation schemes for discrete and continuous land cover classification using decision tree-based algorithms. Remote Sens. 2015, 7, 9655-9681. [CrossRef]

69. Ruder, S. An overview of gradient descent optimization algorithms. arXiv 2016, arXiv:1609.04747.

70. Glorot, X.; Bordes, A.; Bengio, Y. Deep sparse rectifier neural networks. In Proceedings of the 14th International Conference on Artificial Intelligence and Statistics, Fort Lauderdale, FL, USA, 11-13 April 2011; pp. 315-323.

71. LeCun, Y.; Bottou, L.; Bengio, Y.; Haffner, P. Gradient-based learning applied to document recognition. Proc. IEEE 1998, 86, 2278-2324. [CrossRef]

72. He, K.; Zhang, X.; Ren, S.; Sun, J. Deep residual learning for image recognition. In Proceedings of the 29th IEEE Conference on Computer Vision and Pattern Recognition, Las Vegas, NV, USA, 27-30 June 2016; pp. 770-778.

73. Ioffe, S.; Szegedy, C. Batch normalization: Accelerating deep network training by reducing internal covariate shift. arXiv 2015, arXiv:1502.03167.

74. Xie, B.; Zhang, H.K.; Huang, B. Revealing Implicit Assumptions of the Component Substitution Pansharpening Methods. Remote Sens. 2017, 9, 443. [CrossRef]

75. Vivone, G.; Alparone, L.; Chanussot, J.; Mura, M.D.; Garzelli, A.; Licciardi, G.A.; Restaino, R.; Wald, L. A critical comparison among pansharpening algorithms. IEEE Trans. Geosci. Remote Sens. 2015, 53, 2565-2586. [CrossRef]

76. Aiazzi, B.; Baronti, S.; Selva, M. Improving component substitution pansharpening through multivariate regression of MS plus Pan data. IEEE Trans. Geosci. Remote Sens. 2007, 45, 3230-3239. [CrossRef]

77. Garzelli, A. A review of image fusion algorithms based on the super-resolution paradigm. Remote Sens. 2016, 8, 797. [CrossRef]

78. Pan, X.; Zhao, J. High-Resolution Remote Sensing Image Classification Method Based on Convolutional Neural Network and Restricted Conditional Random Field. Remote Sens. 2018, 10, 920. [CrossRef]

79. Zhang, G.; Zhang, R.; Zhou, G.; Jia, X. Hierarchical spatial features learning with deep CNNs for very high-resolution remote sensing image classification. Int. J. Remote Sens. 2018, 39, 5978-5996. [CrossRef] 
80. Liu, T.; Abd-Elrahman, A. An Object-Based Image Analysis Method for Enhancing Classification of Land Covers Using Fully Convolutional Networks and Multi-View Images of Small Unmanned Aerial System. Remote Sens. 2018, 10, 457. [CrossRef]

81. Huang, B.; Zhao, B.; Song, Y. Urban land-use mapping using a deep convolutional neural network with high spatial resolution multispectral remote sensing imagery. Remote Sens. Environ. 2018, 214, 73-86. [CrossRef]

82. Volpi, M.; Tuia, D. Dense semantic labeling of subdecimeter resolution images with convolutional neural networks. IEEE Trans. Geosci. Remote Sens. 2017, 55, 881-893. [CrossRef]

83. Maggiori, E.; Tarabalka, Y.; Charpiat, G.; Alliez, P. Convolutional neural networks for large-scale remote-sensing image classification. IEEE Trans. Geosci. Remote Sens. 2017, 55, 645-657. [CrossRef]

84. Chai, D.; Newsam, S.; Zhang, H.K.; Qiu, Y.; Huang, J. Cloud and cloud shadow detection in Landsat imagery based on deep convolutional neural networks. Remote Sens. Environ. 2019, 225, 307-316. [CrossRef]

(C) 2019 by the authors. Licensee MDPI, Basel, Switzerland. This article is an open access article distributed under the terms and conditions of the Creative Commons Attribution (CC BY) license (http://creativecommons.org/licenses/by/4.0/). 